\title{
Multivariate improved weighted multiscale permutation entropy and its application on EEG data
}

\author{
Mohamad El Sayed Hussein Jomaa ${ }^{\mathrm{a}, *}$, Patrick Van Bogaert ${ }^{\mathrm{a}, \mathrm{b}}$, Nisrine Jrad ${ }^{\mathrm{a}, \mathrm{c}}$, \\ Navah Ester Kadish ${ }^{\mathrm{d}, \mathrm{e}}$, Natia Japaridze ${ }^{\mathrm{e}}$, Michael Siniatchkin ${ }^{\mathrm{d}}$, \\ Marcelo A. Colominas ${ }^{a}$, Anne Humeau-Heurtier ${ }^{a}$ \\ a Univ Angers, LARIS - Laboratoire Angevin de Recherche en Ingénierie des Systèmes, 62 avenue Notre-Dame du Lac, 49000 Angers, France \\ ${ }^{\mathrm{b}}$ Department of Pediatric Neurology, CHU Angers, France \\ c Université Catholique de l'Ouest, Angers-Nantes, France \\ ${ }^{\mathrm{d}}$ Institute of Medical Psychology and Medical Sociology, Christian-Albrecht-University of Kiel, Kiel, Germany \\ e University Hospital of Pediatric Neurology, Kiel, Germany
}

\begin{abstract}
This paper introduces an entropy based method that measures complexity in non-stationary multivariate signals. This method, called Mutivariate Improved Weighted Multiscale Permutation Entropy (mvIWMPE), has two main advantages: (i) it shows lower variance for the results when applied on a wide range of multivariate signals; (ii) it has good accuracy quantifying complexity of different recorded states in signals and hence discriminating them. mvIWMPE is based on two previously introduced permutation entropy algorithms, Improved Multiscale Permutation Entropy (IMPE) and Multivariate Weighted Multiscale Permutation Entropy (mvWMPE). It combines the concept of coarse graining from IMPE and the introduction of the weight of amplitudes of the signals from mvWMPE. mvIWMPE was validated on both synthetic and human electroencephalographic (EEG) signals. Several synthetic signals were simulated: mixtures of white Gaussian noise (WGN) and pink noise, chaotic and convergent Lorenz system signals, stochastic and deterministic signals. As for real signals, resting-state EEG recorded in healthy and epileptic children during eyes closed and eyes open sessions were analyzed. Our method was compared to multivariate multiscale, multivariate weighted multiscale and multivariate improved multiscale permutation entropy methods. Performance on synthetic as well as on EEG signals showed more undeviating results and higher ability for mvIWMPE discriminating different states of signals (chaotic vs convergent, WGN $v s$ pink noise, stochastic $v s$ deterministic simulated signals, and eyes open $v s$ eyes closed EEG signals). We herein proposed an efficient method to measure the complexity of multivariate non-stationary signals. Experimental results showed the accuracy and the robustness (in terms of variance) of the method.
\end{abstract}

\section{Introduction}

Studying signals of living systems is an important step towards better understanding the physiological and pathological functioning of these systems. This is particularly relevant when studying brain signals from electroencephalography (EEG). It is widespread in studies to apply various complexity measuring methods (including multiscale and multivariate) on EEG signals. Examples of that are [1-4]. Indeed, many studies reported a difference in terms of complexity between brain signals from healthy subjects and vari-

\footnotetext{
* Corresponding author.

E-mail address: mohamad.elsayedhusseinjomaa@etud.univ-angers.fr (M. El Sayed Hussein Jomaa).
}

ous pathological conditions [5,6,7], and even between two different physiological states for a given controlled system [8]. However, quantifying the complexity of such signals is a very challenging task because of their inherent properties. First, living systems' signals are non-stationary, and sometimes multivariate, reflecting various underlying mechanisms. Second, these signals disclose a wide diversity caused by the intra- and inter-subject variabilities. In this context, our goal was also to obtain an accurate method to distinguish different physiological states.

A common method for studying irregularity or complexity of time series is entropy. When a signal is regular, its entropy value is rather low. Several algorithms have been proposed to study entropy, such as the approximate entropy [9], sample entropy [10], fuzzy entropy [11], and permutation entropy (PE) [12]. 
PE is based on the concept of ordering the values of the signal being studied. It is characterized in being simple in terms of parameters and fast to compute. For this reason, several studies involved permutation entropy for its advantages [13-18]. Many of these studies were EEG- and epilepsy-oriented as some of them used PE to detect epilepsy and predict seizures [14,16].

PE basically can only be used to find entropy values of signals on a single scale. In 2002, the concept of multiscale entropy was introduced [19]. It is based on "coarse-graining" the original signal to inspect deeper temporal scales and thus calculate entropy values across scales. This multiscale concept was extended to PE. Multiscale permutation entropy was also used in several studies, mainly on physiological signals $[20,21]$. To deal better with physiological signals of systems, several approaches were proposed to extend entropy measures to multivariate signals $[22,23]$ measuring within- and cross-channels dependencies. In 2012, multiscale PE was extended to be applied on multivariate EEG signals and analyse their complexity in the case of Alzheimer's disease [24].

These advances in entropy measurements still have some limitations: (i) they are not robust to signals variabilities and (ii) they show low performance discriminating different physiological or pathological states because they lack information related to signals' amplitudes. The objective of this paper was to introduce a new method that can cope up with all the aforementioned shortcomings. In other terms, a multivariate (across channels) multiscale (across time series) permutation entropy with highly robust results and accurate discrimination ability is here proposed. The method is called multivariate Improved Weighted Multiscale Permutation Entropy (mvIWMPE). This method is validated on both simulated and human EEG signals. As compared to the state of the art methods, it shows higher performance in terms of robustness and discriminability.

This paper is divided into four sections. The first section displays a background on several algorithms of permutation entropy. The second section introduces our new algorithm. The third section evaluates this algorithm on synthetic time series. Finally in the last section, the algorithm is applied on EEG signals to validate its efficiency on real physiological data.

\section{Algorithms of permutation entropy}

In this section, we will briefly describe the different algorithms of PE.

\subsection{Permutation entropy}

Consider a time series $\left\{x_{i}\right\}_{i=1, \ldots, N}$ with $N$ time points. For each time point a vector containing $d$ points is constructed such that $\mathbf{x}_{t}^{d, l}=\left\{x_{t}, x_{t+l}, \ldots, x_{t+(d-2) l}, x_{t+(d-1) l}\right\}$ where $d$ is the embedding dimension and $l$ is the time delay.

Each element of the vector $\mathbf{x}_{t}^{d, l}$ is associated to a number from 1 to $d$ and then ordered in increasing order to become $\left\{x_{t+\left(j_{1}-1\right) l}, x_{t+\left(j_{2}-1\right) l}, \ldots, x_{t+\left(j_{d-1}-1\right) l}, x_{t+\left(j_{d}-1\right) l}\right\} \quad$ with $x_{t+\left(j_{n}-1\right) l} \leq x_{t+\left(j_{n+1}-1\right) l}$. Since the vectors have $d$ points there will be $d$ ! possible orders, $\pi$, named motifs. The relative frequency of each motif, $\pi_{i}$, is calculated as follows

$p\left(\pi_{i}\right)=\frac{\#\left\{t \mid t \leq N-d, \text { type }\left(\mathbf{x}_{t}^{d, l}\right)=\pi_{i}\right\}}{N-d+1}$,

which means the number of all vectors $\mathbf{x}_{t}^{d, l}$ across all $t$ that, when ordered, have the same type as $\pi_{i}$ divided by the total number of vectors $\mathbf{x}_{t}^{d, l}$ (\# means number of elements in a set or cardinality).
Note that the condition $(d+1) ! \leq N$ should be satisfied to get valid entropy results. $\mathrm{PE}$ is then calculated as [12]

$P E_{X}^{d, l}=-\sum_{i=1}^{d !} p\left(\pi_{i}\right) \ln \left(p\left(\pi_{i}\right)\right)$,

where $0 \ln (0)=0$ is set as a convention. This algorithm is based on the original concept of entropy, Shannon Entropy [25], having the same equation.

The higher the value of $d$, the more data is being sorted and the number of motifs increase in a factorial manner. This could lead to more precise results but it will also lead to longer calculation times and would require longer signals. Thus, in order to compensate between the amount of data to be sorted and the calculation time, $d=3$ is commonly used. The value of $l$ is commonly used as 1 [12] as the general case is the intention to study every consecutive time point of the time series without skipping points.

The entropy value is maximum when the time series is totally uncorrelated. In this case, the value of entropy is $\ln (d !)$. This is when the time series is the most irregular. On the other hand, the value of entropy reaches the minimum when the time series is monotonic. The corresponding value is $\ln (1)=0$. As expected, this is when the time series is the least irregular.

\subsection{Multiscale permutation entropy}

Multiscale permutation entropy (MPE) is similar to permutation entropy but includes an additional step: the coarse-graining process [24].

Consider a time series $\left\{x_{i}\right\}_{i=1, \ldots, N}$ with $N$ time points. The coarsegrained time series will be as follows

$y_{j}^{\tau}=\frac{1}{\tau} \sum_{i=(j-1) \tau+1}^{j \tau} x_{i}$,

where $1 \leq j \leq\left\lfloor\frac{N}{\tau}\right\rfloor$. $\tau$ stands for time scale and could range from 1 to $\left\lfloor\frac{N}{(d+1) !}\right\rfloor$. For each time scale $\tau$, PE of the coarse-grained time series is calculated as mentioned in Section 2.1 ending with $P E_{\tau}^{d, l}$. By this way we obtain the value of entropy as a function of time scale.

\subsection{Improved Multiscale Permutation Entropy}

Improved multiscale permutation entropy (IMPE) was introduced by Azami and Escudero [26] to overcome some problems of MPE. These problems are the non-symmetry of the coarse-grained time series and the variability of the MPE results in large time scales.

IMPE is calculated in two steps [26]:

1. For each time scale $\tau, \tau$ different coarse-grained time series are extracted from the original time series. The procedure is as follows

$y_{j}^{k, \tau}=\frac{1}{\tau} \sum_{i=(j-1) \tau+k}^{j \tau+k-1} x_{i}$,

where $1 \leq j \leq\left\lfloor\frac{N}{\tau}\right\rfloor$ and $1 \leq k \leq \tau$.

2. For each $k$, PE of $\mathbf{y}^{k, \tau}$ is calculated such that we obtain $P E_{k, \tau}^{d, l}$. Then IMPE of a certain time scale, $\tau$, would be the average of all the values of $P E_{k, \tau}^{d, l}$ across all $k$

$\operatorname{IMPE}_{(\tau)}^{d, l}=\frac{1}{\tau} \sum_{k=1}^{\tau} P E_{k, \tau}^{d, l}$. 


\subsection{Multivariate multiscale permutation entropy}

Consider a multivariate time series $\left\{x_{m, i}\right\}_{i=1, \ldots, N}$ with $N$ time points, $M$ channels and $m$ representing the channel number $(1 \leq m \leq M)$. For each time scale, $\tau$, the coarse-graining process mentioned in Section 2.2 is applied on each channel. The vectors $\mathbf{x}_{m, t}^{d, l}=\left\{x_{m, t}, x_{m, t+l}, \ldots, x_{m, t+(d-2) l}, x_{m, t+(d-1) l}\right\}$ are extracted for every channel. The elements of $\mathbf{x}_{m, t}^{d, l}$ are ordered and compared with the $d$ ! possible orders or motifs, $\pi_{m}$. The relative frequency of the permutation in each channel is calculated as follows [24]

$p\left(\pi_{m, i}\right)=\frac{\#\left\{t \mid t \leq N-d, \text { type }\left(\mathbf{x}_{m, t}^{d, l}\right)=\pi_{m, i}\right\}}{(N-d+1) M}$.

Note that the relative frequency is divided here by the number of channels, $M$, to maintain $\sum_{m=1}^{M} \sum_{i=1}^{d !} p\left(\pi_{m, i}\right)=1$.

Then the marginal relative frequency of the motifs is calculated

$p\left(\pi_{i}\right)=\sum_{m=1}^{M} p\left(\pi_{m, i}\right)$

So the multivariate multiscale permutation entropy (mvMPE) is calculated [24]

$\operatorname{mvMPE} E_{(\tau)}^{d, l}=-\sum_{i=1}^{d !} p\left(\pi_{i}\right) \ln \left(p\left(\pi_{i}\right)\right)$.

\subsection{Multivariate multiscale weighted permutation entropy}

Multivariate multiscale weighted permutation entropy (mvMWPE) [6] is based on the algorithm of the weighted permutation entropy that was introduced by Fadlallah et al. [27]. This algorithm has the advantage of including the weight of the amplitudes for each permutation.

The steps to calculate mvMWPE are similar to those mentioned in Section 2.4. The coarse-grained time series of the multivariate time series is calculated for a certain scale factor, $\tau$. Then the vectors $\mathbf{x}_{m, t}^{d, l}=\left\{x_{m, t}, x_{m, t+l}, \ldots, x_{m, t+(d-2) l}, x_{m, t+(d-1) l}\right\}$ are extracted for every channel. The elements of these vectors are ordered and compared with the $d$ ! possible motifs, $\pi_{m}$. The absolute frequency of each motif of every channel is then [6]

$p\left(\pi_{m, i}\right)=\sum_{j=1}^{N-d} 1_{v: \operatorname{type}(v)=\pi_{m, i}}\left(\mathbf{x}_{m, j}^{d, l}\right) w_{j}$,

where $1_{A(v)}$ is an indicator function of the set $A$ and is defined as $1_{A(v)}=0$ if $v \notin A$ and $1_{A(v)}=1$ if $v \in A$. $w_{j}$ is the weighted value of the vector $\mathbf{x}_{m, j}^{d, l}$, and is commonly taken as the variance of $\mathbf{x}_{m, j}^{d, l}$.

This would result in a matrix $M \times d$ !, where $M$ is the number of channels. The relative frequency, $p\left(\pi_{m, i}^{d, l}\right)$ of each motif will be the absolute frequency divided by the sum of the matrix. Then the marginal frequency is [6]

$p\left(\pi_{i}\right)=\sum_{m=1}^{M} p\left(\pi_{m, i}\right)$

Finally the mvMWPE is calculated [6]

$\operatorname{mvMWPE} E_{(\tau)}^{d, l}=-\sum_{i=1}^{d !} p\left(\pi_{i}\right) \ln \left(p\left(\pi_{i}\right)\right)$.

\section{Multivariate improved weighted multiscale permutation entropy}

Improved Permutation Entropy proved to have more robust results, in terms of variance, than other PE algorithms due to the averaging of $\tau$ entropy values of each time scale, but it lacks the concept of considering amplitudes of the signals [26]. Moreover, Weighted Permutation Entropy considers the amplitudes of the signals through the addition of the values of variance instead of incrementing the permutation count, but on the other hand it lacks the low variance in results of the Improved Permutation Entropy [6,27].

We therefore introduce Multivariate Improved Weighted Multiscale Permutation Entropy (mvIWMPE) that combines the advantages of both mvMWPE and IMPE, thus covering the drawbacks that were mentioned for each algorithm.

Hence, the steps for calculating mvIWMPE are as follows.

Considering the multivariate time series $\left\{x_{m, i}\right\}_{i=1, \ldots, N}$ with $N$ time points and $M$ channels where $m$ represents the channel number $(1 \leq m \leq M)$ :

1. For each time scale $\tau, \tau$ different coarse-grained time series are extracted for each channel

$y_{m, j}^{k, \tau}=\frac{1}{\tau} \sum_{i=(j-1) \tau+k}^{j \tau+k-1} x_{m, i}$,

where $1 \leq j \leq\left\lfloor\frac{N}{\tau}\right\rfloor$ and $1 \leq k \leq \tau$.

So,

$$
\mathbf{Y}^{k, \tau}=\left[\begin{array}{ccccc}
y_{1,1}^{k, \tau} & y_{1,2}^{k, \tau} & y_{1,3}^{k, \tau} & \ldots & y^{k, \tau} \\
y_{2,1}^{k, \tau} & y_{2,2}^{k, \tau} & y_{2,3}^{k, \tau} & \ldots & \left.y^{k, \tau} \frac{N}{\tau}\right\rfloor \\
& & & & 2,\left\llcorner\frac{N}{\tau}\right\rfloor \\
\ldots & \ldots & \ldots & \ldots & \ldots \\
y_{M, 1}^{k, \tau} & y_{M, 2}^{k, \tau} & y_{M, 3}^{k, \tau} & \ldots & y^{k, \tau} \\
& & & & M,\left\llcorner\frac{N}{\tau}\right\rfloor
\end{array}\right] .
$$

2. For each $k$, mvMWPE of $\mathbf{Y}^{k, \tau}$ is calculated as mentioned in section 2.5 to obtain $m v W M P E_{k, \tau}^{d, l}$

3. Then mvIWMPE at a certain time scale $\tau$ will be calculated as the average of all $m v W M P E_{d, l}^{k, \tau}$ across $k$

$$
\operatorname{mvIWMPE} E_{(\tau)}^{d, l}=\frac{1}{\tau} \sum_{k=1}^{\tau} m v W M P E_{k, \tau}^{d, l} .
$$

The MATLAB script for computing mvIWMPE is available upon request.

\section{Validation on synthetic data}

The performance of mvIWMPE was evaluated with synthetic data using white and pink noise, chaotic signals generated from Lorenz system, and deterministic signals from MIX process. The results were compared with those of MvMPE, MvMWPE, and mVIMPE.

\subsection{White and pink noise}

The first test was the application of all the previously mentioned algorithms on multivariate noise signals. Two types of noise signals were used. The first one is the white Gaussian noise (WGN). The second one is the pink noise, which was generated from WGN by dividing the Fourier transform of the WGN by $\sqrt{f}$, thus the power spectrum of the resulting noise would be proportional to $\frac{1}{f}$. 

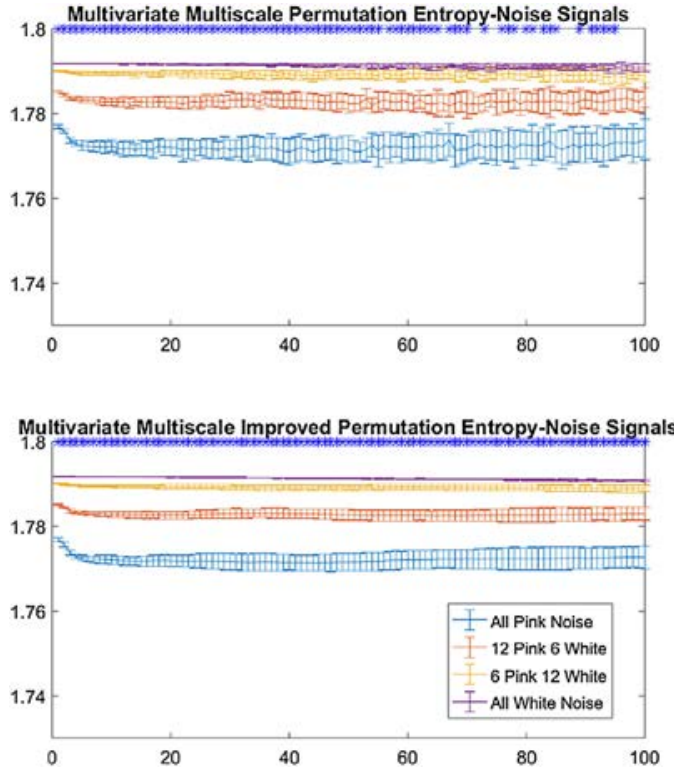
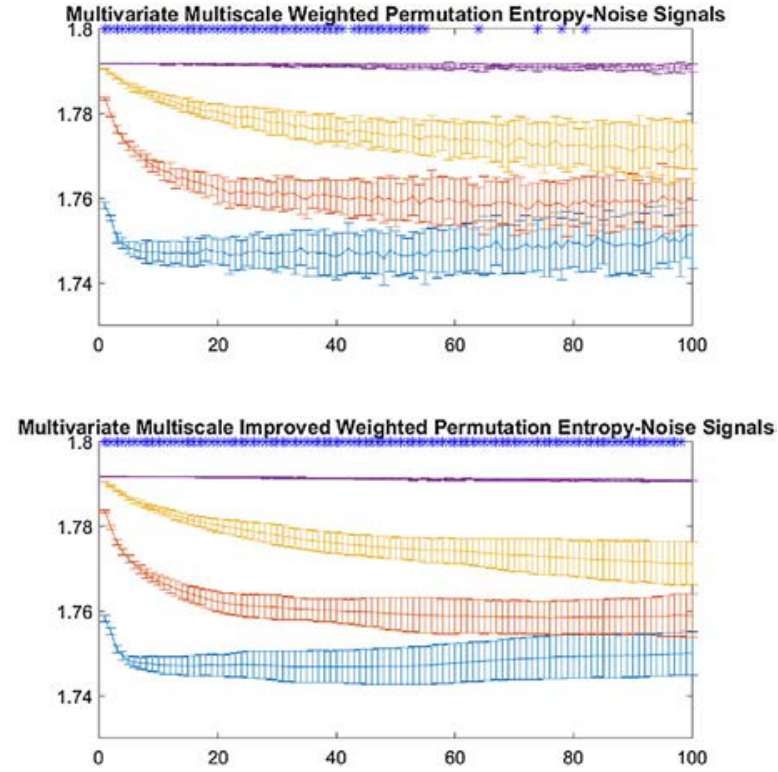

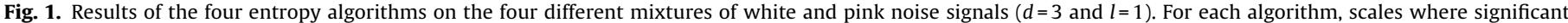
differences (based on Friedman test with Bonferroni correction $p<0.01$ ) appeared between the results of the four noise mixtures were marked with '*'.

All the generated noise signals had 18 channels and 10000 time points, and were made according to four different mixtures:

1. 18 white noise signals

2. 12 white noise signals and 6 pink noise signals

3. 6 white noise signals and 12 pink noise signals

4. 18 pink noise signals

Each group had 30 different realizations. Each of the four algorithms of permutation entropy (mvMPE, mvIMPE, mvMWPE, mvIWMPE) was applied on each of them. The time scales ranged between 1 and 100. To test our method, we used $l=1$ following Brandt's and Pompe's work [12]. In order to respect the condition of $(d+1) ! \leq\left\lfloor\frac{N}{\tau_{\max }}\right\rfloor$, where $N=10000$ and $\tau_{\max }=100$, the highest embedding dimension value was $d=3$. The means and standard deviations of the resulting entropy values were calculated for each group and are presented in Fig. 1.

To evaluate the methods, Friedman tests were applied on pairs of results of different signal mixtures for each method. Those that showed $p$-values less than 0.01 with Bonferroni correction where considered to be significantly different. Fig. 1 marks by '*', for each method, the scales where the differences were significant between the entropy values of all pairs of the 4 mixtures of signals. mvIMPE shows that all 100 scales had significant differences. mvIWMPE shows similar results with 98 scales having significant differences. Those two methods, in comparison with MvMPE and mvMWPE that show lower number of scales with significant differences, show better performances. Those results show that mvIWMPE outperforms mvMPE and mvMWPE and does not ruin the results of mvIMPE when applied on noise signals.

\subsection{Influence of number of channels}

In this test, the effect of increasing the number of channels or variates of the signals is studied. Nine groups of multivariate WGN with 1000 samples were studied. The number of channels ranged between 2 and 10 channels. Each group had 30 realizations. mvIMPE was applied on those groups with $d=3$ and $l=1$. Fig. 2 shows that, as the number of channels increases, the values of entropy change become less decreasing and more constant for WGN across scales. This is due to the fact that the increase of number of channels creates more sample vectors to be sorted and distributed across motifs, thus behaving in a manner similar to finding the multiscale entropy of a univariate signal of the concatenated signals of the channels.

This is supported by the results of Fig. 3. mvIWMPE with $d=3$ and $l=1$ was applied on 2 groups of WGN, one having a dimension of $1 \times 4000$ and the other $4 \times 1000$. This way both groups had the same number of samples (4000). Each group had 30 realizations. The results showed that the two groups have similar values of entropy across scales thus signifying that the two groups are being considered as almost the same signal as if the 4 channels are being concatenated to form a univariate signal.

\subsection{Lorenz system}

The Lorenz system [28] is a system of three differential equations that could result in either chaotic or convergent signals based on the parameters. Its equations are

$\left\{\begin{array}{l}\frac{d x}{d t}=\sigma(y-x) \\ \frac{d y}{d t}=x(\rho-z)-y \\ \frac{d z}{d t}=x y-\beta z\end{array}\right.$.

In our study we fixed the values of $\sigma=10$ and $\beta=\frac{8}{3}$ and used different values of $\rho$ to get different tri-variate chaotic and converging signals. Twenty different values for $0 \leq \rho \leq 1$ resulted in twenty different converging tri-variate signals and twenty different values for $23 \leq \rho \leq 33$ resulted in twenty different chaotic tri-variate signals [29]. The initial point was $(0,5,10)$. All the signals were made up of 10000 time points.

The four permutation entropy algorithms were applied on these signals. The same parameters were used $(d=3$ and $l=1)$. The results in Fig. 4 show that both mvMPE and mvIMPE were not able to differentiate between convergent and chaotic signals. However, the two weighted algorithms showed high ability to differentiate between the two types of signals. But when comparing mvMWPE with mvIWMPE, it is noticed that mvIWMPE has lower standard deviation value that reaches 0 for time scales greater than 60 , not to mention 


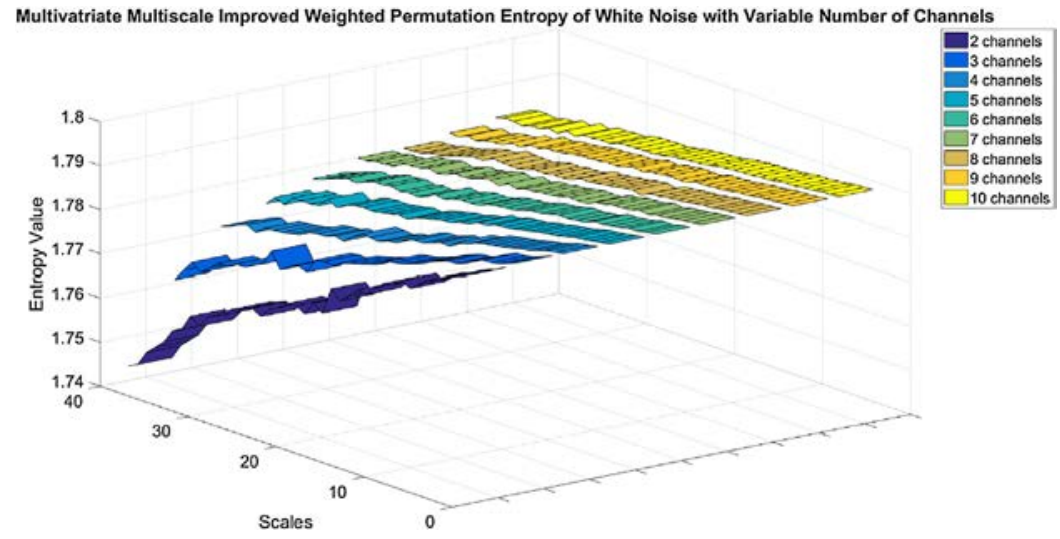

Fig. 2. Results of IWPE on WGN multivariate signals with different number of channels ranging from 2 to $10(d=3$ and $l=1)$.

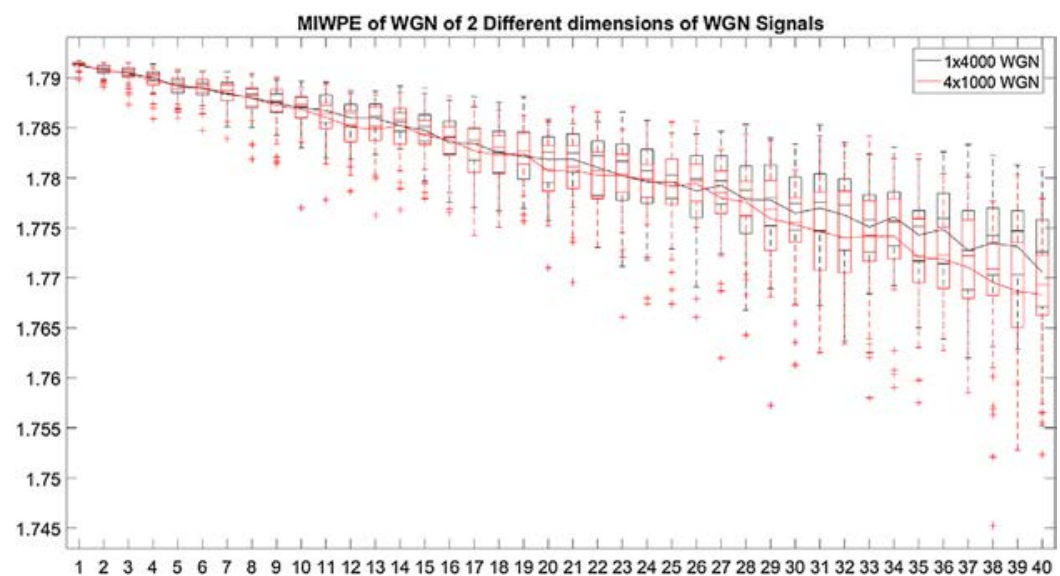

Fig. 3. Results of IWPE on $1 \times 4000$ and $4 \times 1000$ WGN signals $(d=3$ and $l=1)$.

(a)
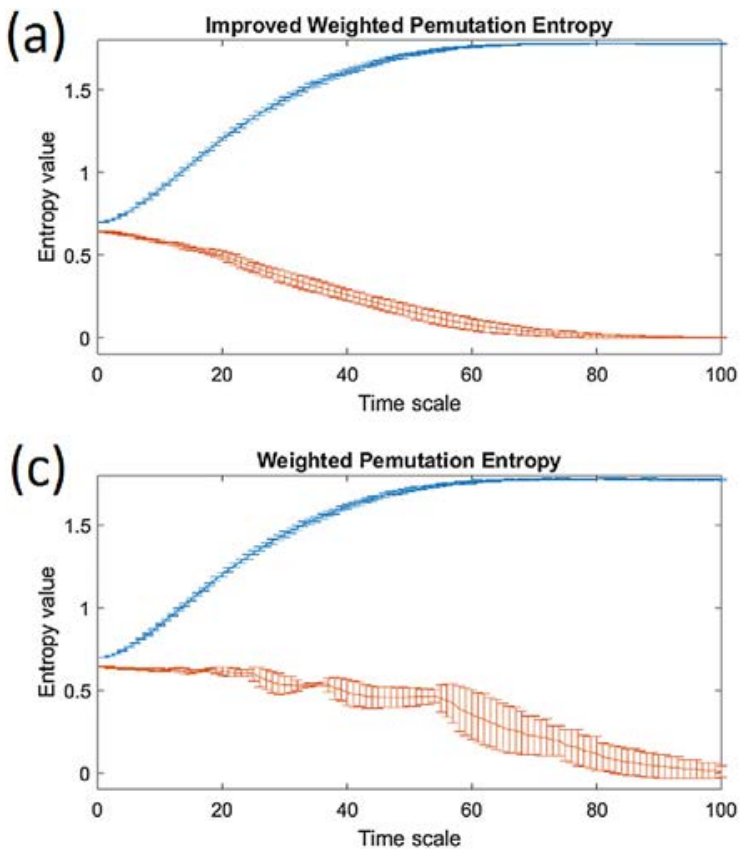

(b) Improved Pemutation Entropy
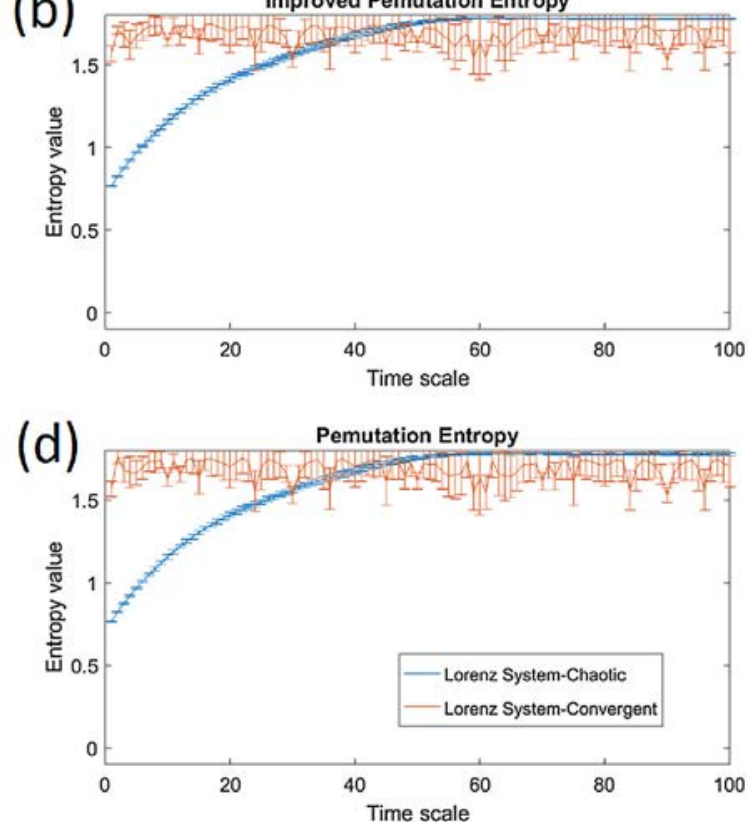

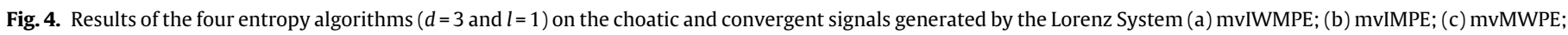
(d) MvMPE. 
(a)

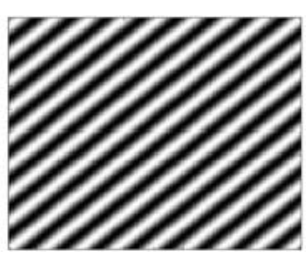

(b)

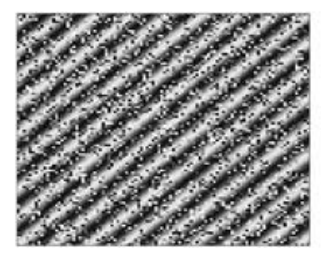

(c)

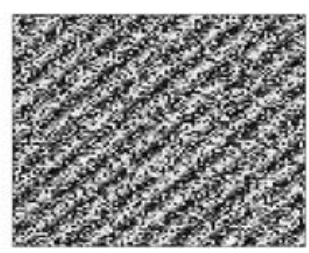

(d)

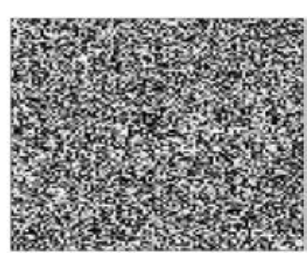

Fig. 5. The generated MIX process: bidimensional $(100 \times 100)$ signals with different values of $p$ (a) $p=0$; (b) $p=0.3 ;(c) p=0.6$; (d) $p=1$.

the smoother curve of the mvIWMPE on converging signals. These results, in addition to the previous ones of noise signals (Section 4.1), show that mvIWMPE outperforms other methods in differentiating between convergent and chaotic signals with smoother curves across scales. mvIWMPE has also similar good performance to mvIMPE in terms of differentiating between different noise signals.

\subsection{MIX process}

The last synthetic test that was applied to evaluate the algorithms was their applications on MIX process signals. This is based on the test introduced by Pincus et al. [9]. In order to test the multivariate algorithm, this test was slightly modified to become two dimensional.

The MIX process is used to generate different levels of stochastic signals that range from being purely deterministic to purely stochastic. To generate such signals, set $p$ as a probability with value between 0 and 1 . Define $X_{m, n}=\alpha^{-1 / 2} \sin \left(\frac{2 \pi}{12}(m+n)\right)$, for $1 \leq m \leq M$ and $1 \leq n \leq N$ with $\alpha$ being the amplitude and defined as

$\alpha=\frac{\sum_{i=1}^{12} \sum_{j=1}^{12} \sin ^{2}\left(\frac{2 \pi}{12}(i+j)\right)}{144}$.

Define $\mathbf{Y}$ as an $M \times N$ matrix of independent identically distributed real random variables having a uniform distribution in the interval $[-\sqrt{3}, \sqrt{3}]$. Define $\mathbf{Z}$ as another $M \times N$ matrix of independent identically distributed real random variables where $Z_{m, n}=1$ with probability $p$ defined earlier and $Z_{m, n}=0$ with probability $1-p$.

Then

$\operatorname{MIX}_{m, n}(p)=\left(1-Z_{m, n}\right) X_{m, n}+Z_{m, n} Y_{m, n}$.

So $\operatorname{MIX}(p)$ would be an $M \times N$ matrix that has $p M N$ of its elements corresponding to a random bi-dimensional signal and $(1-p) M N$ elements corresponding to a periodic bi-dimensional signal. Examples of the MIX signals are shown in Fig. 5.

To test our algorithm, four values of $p$ were used, $p=0.1, p=0.3$, $p=0.6$, and $p=0.9$. For each value, 30 different $18 \times 10000$ signals were generated. Each of the four algorithms of permutation entropy was applied on these signals. The embedding dimension and the lag were unified for all the algorithms as $d=3$ and $l=1$. The time scales ranged between 1 and 100 . The means and standard deviations of the resulting entropy values were calculated for each group and represented as in Fig. 6. The results show that, again, mvIWMPE has the highest differentiation among signals especially for the first ten scales where mvMWPE and mvMPE give very similar results for $p=0.1$ and $p=0.3$. It also shows the highest robustness in terms of variance of entropy results for a given type of signal, which can be confirmed by the smaller error bars.

\section{Evaluation on real EEG signals}

Our new algorithm was also tested on real EEG signals.

\subsection{Dataset 1: healthy children}

Twenty minutes EEG recording from 6 healthy children at rest (four females and two males, age: $8.8 \pm 2.11$ years) were acquired in the Department of Neuropediatrics, ChristianAlbrechts-University, Kiel, Germany. These recordings were approved by the Medical Ethics Committee of the ChristianAlbrechts-University, Kiel, Germany, agreement code “D 460/15".

Subjects were lied down comfortably and rest brain activity was recorded both during eyes open where the subject looked at a fixed picture and eyes closed. The EEG activity was recorded by 128 electrodes (Electrical Geodesics, Inc.) with the Cz electrode as a reference. The sampling rate of all the acquisitions was $1000 \mathrm{~Hz}$. Eyes movements were recorded by two frontal electrodes of the EEG net (E1 and E32) in order to detect drowsiness, and epochs with slow horizontal eyes oscillations were removed from the analysis.

\subsection{Dataset 2: epileptic patients}

The EEG data of 3 epileptic patients recorded in Université Libre de Bruxelles; Hôpital Erasme (agreement of local ethical committee P2015/242) were analyzed. Patient 1 (10 years old male) was diagnosed with epilepsy with CSWS. The epileptic focus was clinically localized on T5 (10-20 system). Patient 2 (8 years old female) was diagnosed with atypical benign childhood epilepsy with centrotemporal spikes (BECTS) with the focus being on C3 (10-20 system). Patient 3 (9 years old male) was diagnosed with BECTS with the focus being on C3 (10-20 system).

All patients had their resting-state EEG recorded while they were lying down for around 20 minutes and alternating between eyes open and eyes closed every minute. The acquisition was done using high density EEG (HD-EEG) with 256 electrodes (Electrical Geodesics, Inc.). The reference was the $\mathrm{Cz}$ electrode and the sampling frequency was $1000 \mathrm{~Hz}$.

\subsubsection{Preprocessing}

The acquired signals were filtered by a bandpass FIR filter of a transition bandwidth of $0.22 \mathrm{~Hz}$ obtained by an order of 15,000 . The cut-off frequencies of the filter were $0.5 \mathrm{~Hz}$ and $45 \mathrm{~Hz}$. Independent component analysis (ICA) [30] was applied on each subject individually to remove artifacts caused by blinking or movement.

For dataset 1, 30 epochs of $10 \mathrm{~s}$ for each case (eyes open and eyes closed) were extracted from each subject. Some of these epochs in some subjects were overlapping (maximum of five seconds overlap).

For dataset 2, 40 non-overlapping epochs of $1 \mathrm{~s}$ for each case (eyes open and eyes closed) were extracted from each subject. The reason for choosing only $1 \mathrm{~s}$ epochs is the appearance of spikes, or interictal epileptiform discharges (IED) and we are interested in epochs without those spikes.

Previous studies showed that the main difference between eyes open and eyes closed in terms of EEG is the presence of the alpha rhythm in the occipital region during the eyes closed case [31]. For this reason, the analysis was performed for that region only. We defined the set of electrodes for each EEG net that covered the 

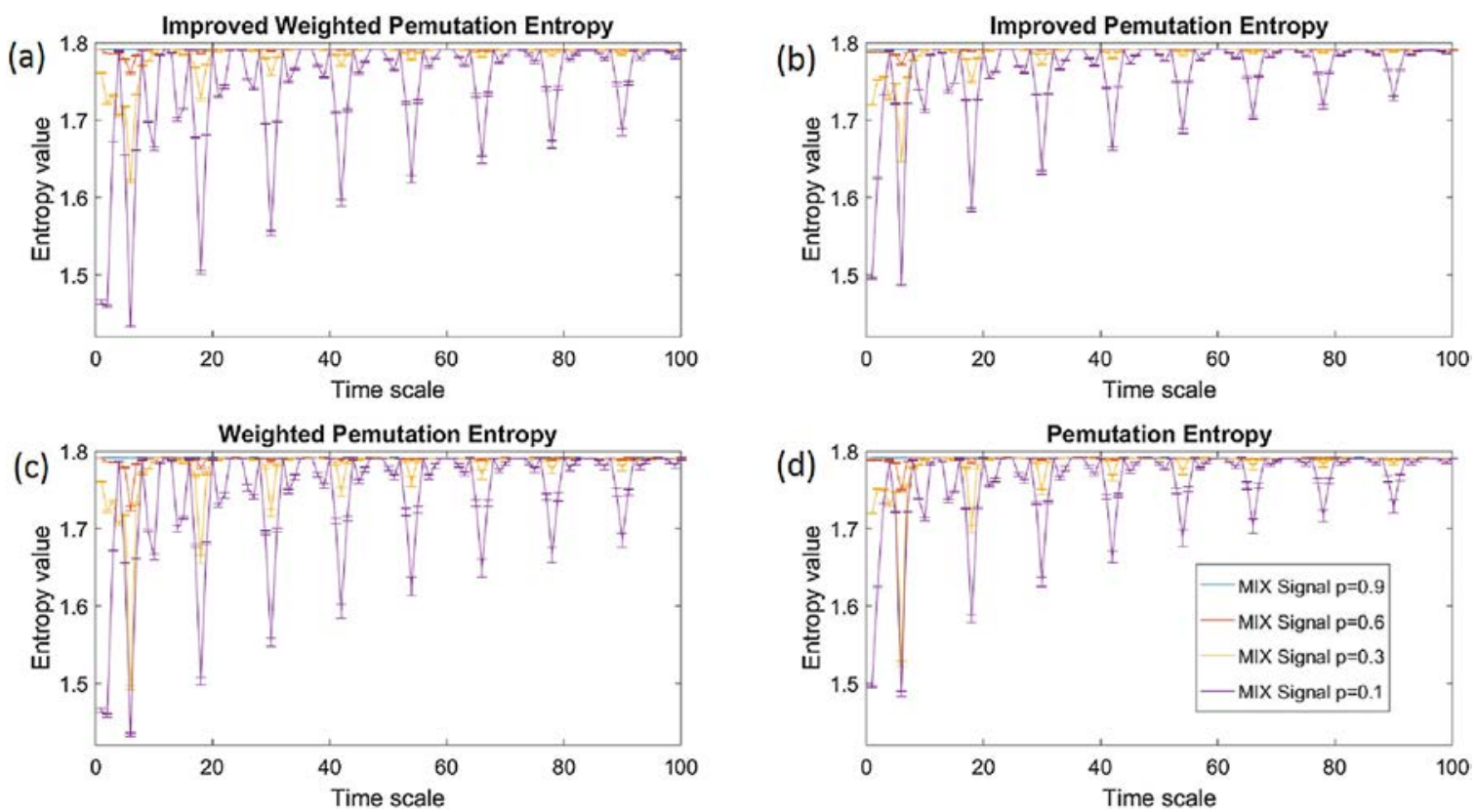

Fig. 6. Results of the four entropy algorithms $(d=3$ and $l=1$ ) on the different MIX process signals generated by 4 different values of p (a) mvIWMPE; (b) mvIMPE; (c) mvMWPE; (d) MvMPE.
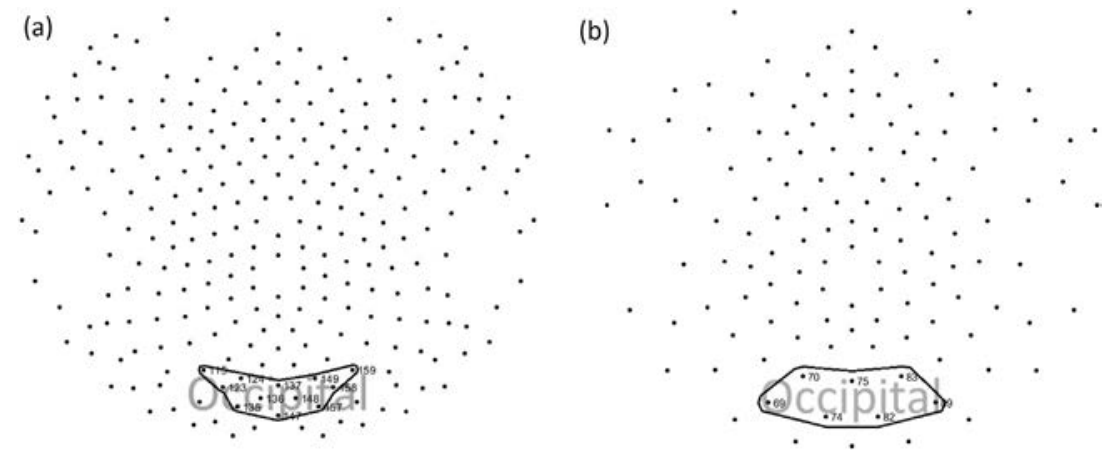

Fig. 7. The occipital region of interest on the (a) 256-channels EEG net and on the (b) 128-channels EEG net.

occipital region with the help of a neurophysiologist. Fig. 7 shows the occipital region for each net used.

All of the preprocessing was done using the EEGLAB [32] toolbox for Matlab R2016b.

\subsection{Results of EEG signals evaluation}

The goal of this evaluation was to see whether mvIWMPE maintains higher ability of differentiation between two states when applied to real EEG signals, which in our case are eyes open and eyes closed. The four multivariate algorithms of permutation entropy were then applied on the epoched signals of each subject in each dataset, with $d=3$ and $l=1$ in a time scale ranging from 1 to 40 .

In general, all the curves had a common form which is similar to that of the chaotic signals of the Lorenz system (a decaying increase of entropy value with respect to time scale) (Fig. 4). However it is worth mentioning that each subject behaved differently in a certain normal range and resulted in different curves that differentiated eyes open from eyes closed entropy values. Thus, averaging the results across subjects would diminish this differentiation for such relatively small number of subjects. For this reason, each subject was studied separately.

The results for all subjects showed that entropy values were lower in the case of eyes closed, for scales less than 30 . This was expected since, as mentioned in Section 5.2.1, the eyes closed state is characterized by the alpha waves that makes the recorded EEG signals more periodic and thus less complex. The reason behind having 30 as the maximum scale where the entropy of eyes closed signal was less than the entropy of eyes open signals is that the coarse-graining window becomes larger than the alpha oscillation at this scale.

For all subjects in both datasets, Friedman tests were applied on the results of the eyes open and eyes closed condition to evaluate whether we have significant differences between the two results. Scales with Bonferroni corrected $p$-values less than 0.01 were considered to be significantly different. Only significant differences of scales less than 30 were considered. Figs. 8 and 9 summarize the results of the Friedman tests for both datasets. Scales labeled in red had the entropy values of eyes open significantly higher than those of eyes closed. The total number of scales in red is given for each subject or patient and for each method in each dataset. For dataset 1, both mvIMPE and mvIWMPE had the highest number of scales in red (151) showing that both have good ability in differentiating between signals of eyes open and eyes closed states. For dataset 2 , mvIWMPE had the highest number of scales in red (25) showing the best ability in differentiating between the two cases of EEG signals.

It worth mentioning that dataset 1 had epochs of 10 seconds while dataset 2 had epochs of 1 second. This is the reason the results 


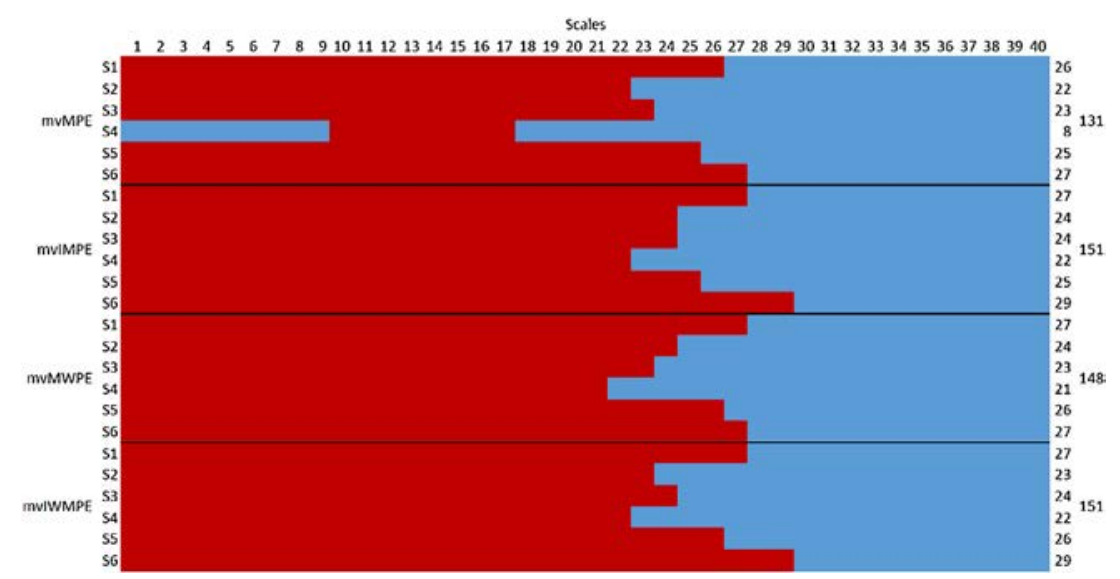

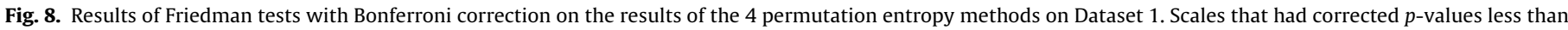

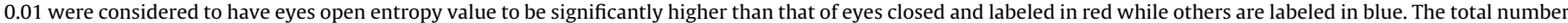
of scales with significant differences for each subject and for each method is given at the end of each row.

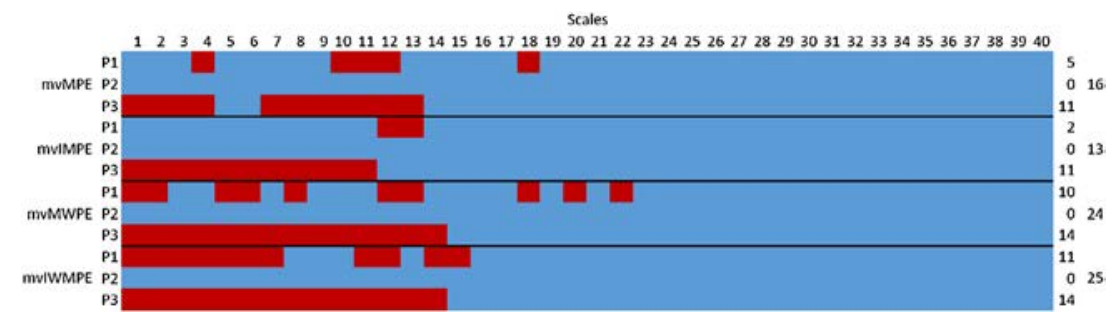

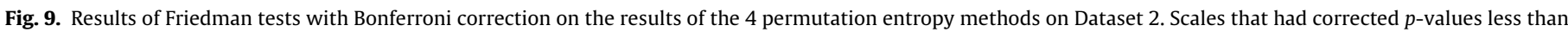

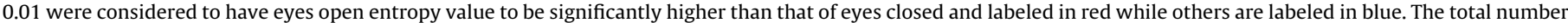
of scales with significant differences for each patient and for each method is given at the end of each row.

of dataset 1 have much higher number of scales in red as for longer signals each method gave more precise results due to the increase in the number of embedding vectors to be grouped into motifs. This also highlights the fact that for shorter signals mvIWMPE shows better performance as shown in Fig. 9.

\section{Conclusion}

We proposed a multiscale permutation entropy-based method to compute irregularity of multivariate complex signals. This method, called mvIWMPE has the advantages of being robust and able to discriminate between different states in a single signal. The efficiency of mvIWMPE was proven in synthetic and human EEG signals.

It would be interesting, in the future, to apply mvIWMPE on EEG signals of patients suffering from neurological diseases to see whether it could be a biomarker of the disease by showing decreased entropy values in patients compared to control subjects. Moreover, this method could contribute to assess the efficacy of a treatment in comparing EEG signals obtained before and after a therapeutic intervention.

\section{Acknowledgement}

This work was supported by the European Commission, Project FP7 DESIRE (Health-F2-602531-2013). M. El Sayed Hussein Jomaa and M. A. Colominas are both full-time granted by the DESIRE project.

\section{References}

[1] I. Rezek, S.J. Roberts, Stochastic complexity measures for physiological signal analysis, IEEE Trans. Biomed. Eng. 45 (9) (1998) 1186-1191.
[2] H. Adeli, et al., A wavelet-chaos methodology for analysis of EEGs and EEC subbands to detect seizure and epilepsy, IEEE Trans. Biomed. Eng. 54 (2) (2007) 205-211.

[3] X. Kang, et al., Multiscale entropy analysis of EEG for assessment of post-cardiac arrest neurological recovery under hypothermia in rats, IEEE Trans. Biomed. Eng. 56 (4) (2009) 1023-1031.

[4] V. Srinivasan, et al., Approximate entropy-based epileptic EEG detection using artificial neural networks, IEEE Trans. Inf. Technol. Biomed. 11 (3) (2007) 288-295.

[5] H. Kantz, et al., Nonlinear Analysis of Physiological Data, Springer Science \& Business Media, 2012.

[6] B. Deng, et al., Multivariate multi-scale weighted permutation entropy analysis of EEG complexity for Alzheimer's disease, Cognit. Neurodyn. 11 (2017) 217-231.

[7] M.A. Colominas, M. El Sayed Hussein Jomaa, N. Jrad, A. Humeau-Heurtier, P. Van Bogaert, Time-varying time-frequency complexity measures for epileptic EEG data analysis, IEEE Trans. Biomed. Eng. 65 (8) (2018) 1681-1688.

[8] R.J. Barry, et al., EEG differences between eyes-closed and eyes-open resting conditions, Clin. Neurophysiol. 118 (12) (2007) 2765-2773.

[9] S.M. Pincus, Approximate entropy as a measure of system complexity, Proc. Natl. Acad. Sci. 88 (6) (1991) 2297-2301.

[10] J.S. Richman, J.R. Moorman, Physiological time-series analysis using approximate entropy and sample entropy, Am. J. Physiol. - Heart Circ. Physiol. 278 (6) (2000) H2039-H2049.

[11] W. Chen, et al., Characterization of surface EMG signal based on fuzzy entropy, IEEE Trans. Neural Syst. Rehabil. Eng. 15 (2) (2007) 266-272.

[12] C. Bandt, B. Pompe, Permutation entropy: a natural complexity measure for time series, Phys. Rev. Lett. 88 (17) (2002) 174102.

[13] Y. Cao, W.-W. Tung, J. Gao, V.A. Protopopescu, L.M. Hively, Detecting dynamical changes in time series using the permutation entropy, Phys. Rev. E 70 (4) (2004) 046217.

[14] X. Li, G. Ouyang, D.A. Richards, Predictability analysis of absence seizures with permutation entropy, Epilepsy Res. 77 (1) (2007) 70-74.

[15] M. Zanin, L. Zunino, O.A. Rosso, D. Papo, Permutation entropy and its main biomedical and econophysics applications: a review, Entropy 14 (8) (2012) 1553-1577.

[16] N. Nicolaou, J. Georgiou, Detection of epileptic electroencephalogram based on permutation entropy and support vector machines, Expert Syst. Appl. 39 (1) (2012) 202-209.

[17] E. Olofsen, J. Sleigh, A. Dahan, Permutation entropy of the electroencephalogram: a measure of anaesthetic drug effect, Br. J. Anaesth. 101 (6) (2008) 810-821. 
[18] A.A. Bruzzo, B. Gesierich, M. Santi, C.A. Tassinari, N. Birbaumer, G. Rubboli, Permutation entropy to detect vigilance changes and preictal states from scalp EEG in epileptic patients. A preliminary study, Neurol. Sci. 29 (1) (2008) 3-9.

[19] M. Costa, et al., Multiscale entropy analysis of complex physiologic time series, Phys. Rev. Lett. 89 (6) (2002) 068102.

[20] D. Li, X. Li, Z. Liang, L.J. Voss, J.W. Sleigh, Multiscale permutation entropy analysis of EEG recordings during sevoflurane anesthesia, J. Neural Eng. 7 (4) (2010) 046010

[21] W. Aziz, M. Arif, Multiscale permutation entropy of physiological time series 9th International Multitopic Conference, IEEE INMIC 2005, IEEE (2005) 1-6.

[22] A. Humeau-Heurtier, Multivariate generalized multiscale entropy analysis, Entropy 18 (11) (2016) 411.

[23] M.U. Ahmed, D.P. Mandic, Multivariate multiscale entropy analysis, IEEE Signal Process. Lett. 19 (2) (2012) 91-94

[24] F.C. Morabito, et al., Multivariate multi-scale permutation entropy for complexity analysis of Alzheimer's disease EEG, Entropy 14 (7) (2012) 1186-1202.

[25] C.E. Shannon, A mathematical theory of communication, ACM SIGMOBILE Mobile Comput. Commun. Rev. 5 (1) (2001) 3-55.
[26] H. Azami, J. Escudero, Improved multiscale permutation entropy for biomedical signal analysis: interpretation and application to electroencephalogram recordings, Biomed. Signal Process. Control 23 (2016) $28-41$.

[27] B. Fadlallah, et al., Weighted-permutation entropy: a complexity measure for time series incorporating amplitude information, Phys. Rev. E 87 (2) (2013) 022911.

[28] E.N. Lorenz, Deterministic nonperiodic flow, J. Atmos. Sci. 20 (2) (1963) $130-141$

[29] L.A. Sanchez, Convergence to equilibria in the Lorenz system via monotone methods, J. Differ. Equ. 217 (2) (2005) 341-362.

[30] A. Hyvärinen, et al., Independent Component Analysis, vol. 46, John Wiley \& Sons, 2004.

[31] J.S. Ebersole, T.A. Pedley, Current Practice of Clinical Electroencephalography, Lippincott Williams \& Wilkins, 2003.

[32] A. Delorme, S. Makeig, EEGLAB: an open source toolbox for analysis of single-trial EEG dynamics including independent component analysis, J. Neurosci. Methods 134 (1) (2004) 9-21. 